\title{
Dual inhibition of RNAi therapeutic miR-26a-5p targeting cMet and immunotherapy against EGFR in endometrial cancer treatment
}

\author{
Yun Liü, Yixuan Cai", Yue Chang \\ Department of Obstetrics and Gynecology, Beijing Friendship Hospital Affiliated to Capital Medical University, Beijing, China \\ Contributions: (I) Conception and design: Y Liu, Y Cai; (II) Administrative support: None; (III) Provision of study materials or patients: None; (IV) \\ Collection and assembly of data: Y Cai, Y Chang; (V) Data analysis and interpretation: All authors; (VI) Manuscript writing: All authors; (VII) Final \\ approval of manuscript: All authors. \\ \#These authors contributed equally to this work as co-first authors. \\ Correspondence to: Yun Liu. Department of Obstetrics and Gynecology, Beijing Friendship Hospital Affiliated to Capital Medical University, Beijing, \\ China. Email: liuyun.bjfh@ccmu.edu.cn.
}

Background: Precise prediction of drug combination targeting tumor cells effectively is a crucial challenge for tumor therapy, especially for endometrial cancer (EC). Considering the resistance, crosstalk that occurs between the receptor tyrosine kinase mesenchymal-epithelial transition factor (cMet) and epidermal growth factor receptor (EGFR), and their indispensable influence on the occurrence of EC, this study aimed to explore a novel therapeutic approach for EC treatment through blocking cMet and EGFR simultaneously.

Methods: In the present study, the expression of miR-26a-5p in EC cell lines was detected using quantitative real-time polymerase chain reaction assay. The potential role of miR-26a-5p in the development of EC was examined using cell counting kit assay, 5-ethynyl-2'-deoxyuridine staining, wound healing assay, and cell apoptosis staining assay. Subsequently, the effect of upregulated miR-26a-5p in vivo was confirmed on a xenograft model. Luciferase reporter assay and Western blot analysis were performed to verify the relation between miR-26a-5p and cMet. Furthermore, the dual therapeutic effect of miR-26a-5p and EGFR monoclonal antibody cetuximab was confirmed in vivo and in vitro.

Results: The results indicated that miR-26a-5p expression significantly reduced in EC cell lines compared with the normal endometrial cell line. Furthermore, the overexpression of miR-26a-5p inhibited the progression of EC, including cell migration, cell proliferation, and cell apoptosis in vivo and in vitro. Subsequently, mir-26a-5p regulated the expression of cMet and the downstream the hepatocyte growth factor (HGF)/cMet pathway, thus exerting an inhibitory effect on EC cells. In addition, the study also demonstrated that the upregulation of miR-26a-5p could significantly enhance the inhibitory effect of cetuximab compared with the use of cetuximab alone in vivo and in vitro.

Conclusions: RNAi therapeutic miR-26a-5p suppressed the progression of EC through regulating the cMet/HGF pathway. The dual therapy using RNA interference and neutralizing antibody simultaneously blocked tumor targets, including cMet and EGFR, thus providing a novel approach for overcoming the resistance to the inhibitors against a single target in EC treatment.

Keywords: Cetuximab; mesenchymal-epithelial transition factor (cMet); epidermal growth factor receptor (EGFR); immunotherapy; miR-26a-5p

Submitted Apr 06, 2020. Accepted for publication Oct 10, 2020.

doi: 10.21037/atm-20-3166

View this article at: http://dx.doi.org/10.21037/atm-20-3166 


\section{Introduction}

Endometrial cancer (EC) is the fourth most common cancer in women worldwide with high morbidity and mortality. More than 60,000 women were newly diagnosed with uterine corpus cancer in 2016 in the United States $(1,2)$. Based on histopathological features, most endometrial carcinomas belong to type I tumors, which are often triggered by exogenous and endogenous estrogen exposure. Approximately $20 \%$ of endometrial carcinomas are largely considered to be type II subtypes, which are endocrine independent and preceded by endometrial polyps $(3,4)$. The mainstay of traditional therapy for patients with advanced endometrial carcinoma is surgical treatment combined with radiation and chemotherapy; however, the prognosis of advanced endometrial carcinoma is still poor $(5,6)$. Therefore, the molecular mechanism needs to be understood to explore a new treatment approach.

Tyrosine kinases are considered to be a critical factor in the progression of multiple tumors, including receptors for epidermal growth factor (EGF), vascular endothelial growth factor, and hepatocyte growth factor (HGF), which selectively phosphorylate their substrates to activate downstream signaling pathways (7). Numerous studies reported the aberrant hyperactivation of the HGF/the receptor tyrosine kinase mesenchymal-epithelial transition factor (cMet) pathway in many cancers, including EC, which could promote cancer invasion and metastasis (8-12). As to $\mathrm{HGF} / \mathrm{cMet}$ pathway, it was demonstrated that after the ligand HGF bond to the cMet extracellular domain, the dimerization, autophosphorylation, and activation of cMet was triggered, thus activating downstream pathways. Therefore, HGF/cMet has been considered as a potential therapeutic target. In addition, the overexpression of $\mathrm{cMet}$ is known to be the cause of epidermal growth factor receptor (EGFR)-related drug resistance in clinical trials. EGFR and cMet share overlapping downstream signaling pathways and can trans-phosphorylate one another $(13,14)$. As EGFR activation can contribute to the activation of cMet, the signaling pathways of c-Met and EGFR have a complex interplay. Given the interrelationship between EGFR and c-Met, a combination of cMet target inhibitor with EGFRtyrosine kinase inhibitor (TKIs) has been a therapeutically actionable regimen in clinical treatment $(15,16)$. In EC, several studies have indicated that EGFR was vital in EC and EGFR expression was associated with patient outcomes in $43-67 \%$ of EC tissue samples (17-20). Notably, the cMet/EGFR signal was also found to be implicated in the initiation and progression of EC. Molecular and genetic investigations provided evidence that EGFR correlated with cMet in 69 consecutive tumors and 16 tissue microarrays (21). Collectively, cMet/EGFR inhibition might have a clinical benefit for patients with EC.

Besides immunotherapy against different checkpoints, RNAi therapeutics also have attracted great attention especially after patisiran, the first siRNA-based drug, was approved by the US Food and Drug Administration in 2018. MicroRNAs (miRNAs) are identified as a class of small noncoding RNAs with a role in various biological processes by silencing target genes in the post-transcriptional stage. Previous studies highlighted the connection between cMet and microRNAs. For instance, the upregulation of endogenous miRNAs (miR-34b, miR-34c, and miR-199a), as antagonists of cMet, resulted in the reduced expression of cMET protein and blocked Met-induced signal transduction in tumor cells with hyperactive cMet signa $(22,23)$. Considering the non-negligible effect of miRNAs, Muralidharan performed miRNA profiling in endometrial tissues and normal endometrium, demonstrating that the expression of miR-26 decreased in EC cell lines (24); however, the molecular mechanism of miR-26a-5p was not reported. Based on the available evidence, it was hypothesized that miR-26a-5p might be associated with the progression of EC.

We present the following article in accordance with the ARRIVE reporting checklist (available at http://dx.doi. org/10.21037/atm-20-3166).

\section{Methods}

\section{Cell culture and reagents}

EC cell lines (HEC-1A and RL-952) were purchased from BeNa Culture Collection (Beijing, China). All the cell lines were cultured at $37{ }^{\circ} \mathrm{C}$ with $5 \% \mathrm{CO}_{2}$. The cell medium, including RPMI-1640 and DMEM, were obtained from thermoFisher Company and used for the culture of EC cell lines after mixing with $10 \%$ fetal bovine serum (Gibco, USA). The antibodies used in the study were as follows: anti-cMet (Santa Cruz, USA); Cetuximab (Selleck, China).

\section{Quantitative real-time polymerase chain reaction}

Total RNA was extracted from tissues, and frozen in liquid nitrogen using TRIzol RNA isolation reagent (Invitrogen, USA). First-strand cDNA synthesis was performed using 
a PrimerScript RT Reagent Kit (Takara, Japan) following the manufacturer's protocol. Then, quantitative real-time polymerase chain reaction (qRT-PCR) was used to measure the level of RNA using a Mir-X miRNA qRT-PCR TB Green Kit (TaKaRa, USA). The expression of mRNA and miRNAs was quantified on an ABI 7500 real-time PCR system. $\beta$-Actin was selected as the internal control. The sequences of primers used in the PCR assay were as follows:

$\beta$-actin, 5'-ATAGCACAGCCTGGATAGCAACGTAC$3^{\prime}$ (forward) and 5'-CACCTTCTACAATGAGCTG CGTGTG-3' (reverse);

cMet, 5' -AGCAATGGGGAGTGTAAAGAGG3 (forward) and 5'-CCCAGTCTTGTACTCAGCAAC-3' (reverse);

U6, 5'-CTCGCTTCGGCAGCACA-3' (forward) and 5'-AACGCTTCACGAATTTGCGT-3' (reverse);

miR-26a-5p mimic: 5'-UUCUCCGAACGUGUCAC GUTT-3'.

\section{Cell viability/toxicity assays}

To detect the miR-26a-5p's effect on EC cells, cell proliferation was evaluated using a CCK-8 cell counting kit (Vazyme Biotech, China). HEC-1A cells were subcultured into 96-well plates at a density of 2,500 cells/well after transfection with related plasmids. After culturing the cells for $24 \mathrm{~h}, 10 \mu \mathrm{L}$ of CCK-8 reagent was added to each cell plate and incubated for $2 \mathrm{~h}$ at $37^{\circ} \mathrm{C}$. The absorbance was recorded at a wavelength of $450 \mathrm{~nm}$ using Tecan Infinite Pro (Tecan, Switzerland). For detecting the inhibitory effect of cetuximab, cell toxicity assay was performed using a CellTiter-Glo kit (Promega, USA). The cells were fed into a 96-well plate at a density of 5,000 cells/well. After culturing the cells for $24 \mathrm{~h}$, cetuximab was added into the well diluted in a four-fold gradient at an initial concentration of $20 \mu \mathrm{g} / \mathrm{mL}$. After $72 \mathrm{~h}$, the CellTiter-Glo (CTG) reagent was added to the well and detected using BioTek Cytation (biotek, USA).

Cell death was measured using flow cytometric analysis. After transfection, the cells were collected and resuspended in phosphate buffer saline (PBS). Then, the cells were stained with Annexin-V and propidium iodide (PI, Sigma, USA). The data were collected using fluorescentactivated cell sorting (FACS) CantoII (BD Biosciences, CA, USA) and analyzed using FlowJo software (Tree Star Corp., CA, USA).

\section{5-ethynyl-2'-deoxyuridine assay}

5-ethynyl-2'-deoxyuridine (EdU) was also used to evaluate cell proliferation using a Click-iT EdU Cell Proliferation Kit (ThermoFisher, USA) following the manufacturer's protocols. The cells were seeded into 96-well plates and cultured for $24 \mathrm{~h}$. After transfection, the cells were stained with EdU and 4',6-diamidino-2-phenylindole (DAPI). The number of positively stained cells was calculated using ImageJ software.

\section{Wound bealing assay}

A wound-healing assay was conducted to confirm the cell migration of EC. After transfection, the cells were seeded into six-well plates at a density of 1,000 cells/well. The wounds were created with a $10-\mu \mathrm{L}$ pipette tip. The images were captured using a microscope after 0,12 , and $24 \mathrm{~h}$. The area and distance migrated were measured using ImageJ.

\section{Tumor xenograft model}

All experimental animals were obtained from Southern Model Organisms (Shanghai, China) and housed under specific-pathogen-free conditions. The experiments were performed according to the USA National Institutes of Health guidelines and approved by the local animal care and use committee of Capital Medical University (18-2032). Tumor cells were subcutaneously inoculated into the abdomen of 5-week-old nude mice. After 1 week, the drug or vehicle was injected into the animal model. The tumor volume was calculated using the following formula: $V=$ $1 / 2 a b^{2}$ ( $a=$ long diameter; $b=$ short diameter), and the tumor growth curve was plotted by calculating the tumor volume each week after the injection. Each group comprised more than six female mice.

\section{Immunobistochemical staining}

The tumor samples were sliced into $30-$ to $40-\mu \mathrm{m}$ histologic sections on a histo-cryotome after fixing with $4 \%$ paraformaldehyde, and the tissue slices were dewaxed using dimethylbenzene. Then, the sections were incubated with primary antibody overnight at $4{ }^{\circ} \mathrm{C}$ after antigen retrieval. Further, they were incubated with the secondary antibody at $37^{\circ} \mathrm{C}$ for $2 \mathrm{~h}$. A suitable amount of 3 '-diaminobenzidine tetrahydrochloride $(\mathrm{DAB})$ reagent was added to the sections 
for 10-30 min, and the staining intensity was checked with a bright-field microscope.

\section{Statistical analysis}

All the experiments were performed in at least three independent replicates. The data were analyzed using GraphPad Prism 6.0. The Student's $t$-test or one-way analysis of variance was used for statistical analysis. The $\mathrm{P}$ value $(\mathrm{P} \leq 0.05)$ was considered to indicate a statistically significant difference. Data are presented as mean \pm standard deviation.

\section{Results}

Expression of miR-26a-5p was reduced in EC cells

The expression of miR-26a-5p was determined in 538 uterine corpus endometrial carcinoma (UCEC) samples and 33 normal samples from starBase 3.0 website to confirm the hypothesis that miR-26a-5p had abnormal expression in EC (25). The data showed that miR-26a-5p expression decreased in UCEC samples (Figure 1A). Then, the level of miR-26a-5p was assessed in endometrial cell lines using qRT-PCR. The results showed that the endogenous level of miR-26a-5p was lower in EC cell lines HEC-1A, Ishikawa, RL-952, and JEC compared with normal endometrium cells, suggesting a role of miR-26a-5p in EC cells (Figure $1 B$ ). MiR-26a-5p was transiently overexpressed by transfecting HEC-1A and RL-952 cells with miR-26a-5p mimic and control mimic to identify whether miR-26a-5p functioned in EC tumorigenesis. Then, the efficiency of transfection was evaluated using a qPCR assay (Figure 1C).

\section{Upregulation of miR-26a-5p inbibited cell proliferation and cell migration and promoted the apoptosis of EC cells}

The cell proliferation ability was examined using the cell counting kit 8 (CCK-8) assay after transfection with miR$26 a-5 p$ mimic to directly assess whether miR-26a-5p affected tumor formation in EC cells. The result showed that the overexpression of miR-26a-5p restrained the growth of EC cells (Figure 2A). Furthermore, EdU staining assay was carried out, which could label the cells with DNA synthesis or S-phase synthesis of the cell cycle via click chemistry (26-28). The results were consistent with the findings of CCK-8 assay (Figure 2B,C), suggesting that
miR-26a-5p had an important influence on the proliferation of EC cells. Cell migration ability was also one of the most important characteristics of the occurrence of epithelialmesenchymal transition (EMT) in EC cells. Therefore, the wound healing assay was conducted after transfecting miR-26a-5p mimic and control mimic into EC cells. The migration distance was found to be narrower in EC cells transfected with miR-26a-5p mimic compared with the control (Figure 2D,E), suggesting that the upregulation of miR-26a-5p could inhibit cell migration ability in EC cells. In addition, flow cytometry was used to detect the number of apoptotic cells after transfection with miR-26a-5p mimic. The data showed that miR-26a-5p promoted the death of EC cells in the miR-26a-5p mimic group (Figure 2F,G). Taken together, it was concluded that the overexpression of miR-26a-5p could restrain the progression of EC tumorigenesis, including cell proliferation, cell migration, and cell apoptosis.

\section{MiR-26a-5p had antitumor activity in vivo}

Female nude mice were subcutaneously injected with EC cells transfected with miR-26a-5p mimic and control mimic to establish the xenograft model so as to identify the biological function of miR-26a-5p in vivo. All mice had similar health status prior to treatment. After injection for almost 7 days, the tumor weight and volume were measured once the tumor volume was about $100 \mathrm{~mm}^{3}$. The tumor growth curve indicated that the upregulation of miR-26a-5p retarded the growth of transplanted endometrial tumors (Figure $3 A, B)$. In addition, tumor weight was measured to demonstrate the similar antitumor effect of miR-26a-5p (Figure 3C). Hence, it was concluded that miR-26a-5p was also involved in the formation of EC tumors in vivo. Furthermore, immunohistochemical staining was also conducted to confirm the level of Ki-67, which was used as a marker of cellular proliferation. As shown in Figure 3D,E, the result revealed that the number of Ki67-positive cells was significantly less in the miR-26a-5p mimic group than in the control mimic group, indicating that elevated miR26a-5p expression suppressed cell proliferation in vivo (Figure 3D,E). Moreover, cell apoptosis was also detected in transplanted endometrial tumors using TdT-mediated dUTP nick-end labeling (TUNEL) staining. MiR-26a-5p promoted cell death in vivo (Figure $3 F, G$ ). These results showed that the upregulation of miR-26a-5p had an inhibitory effect on tumor growth in the xenograft model. 

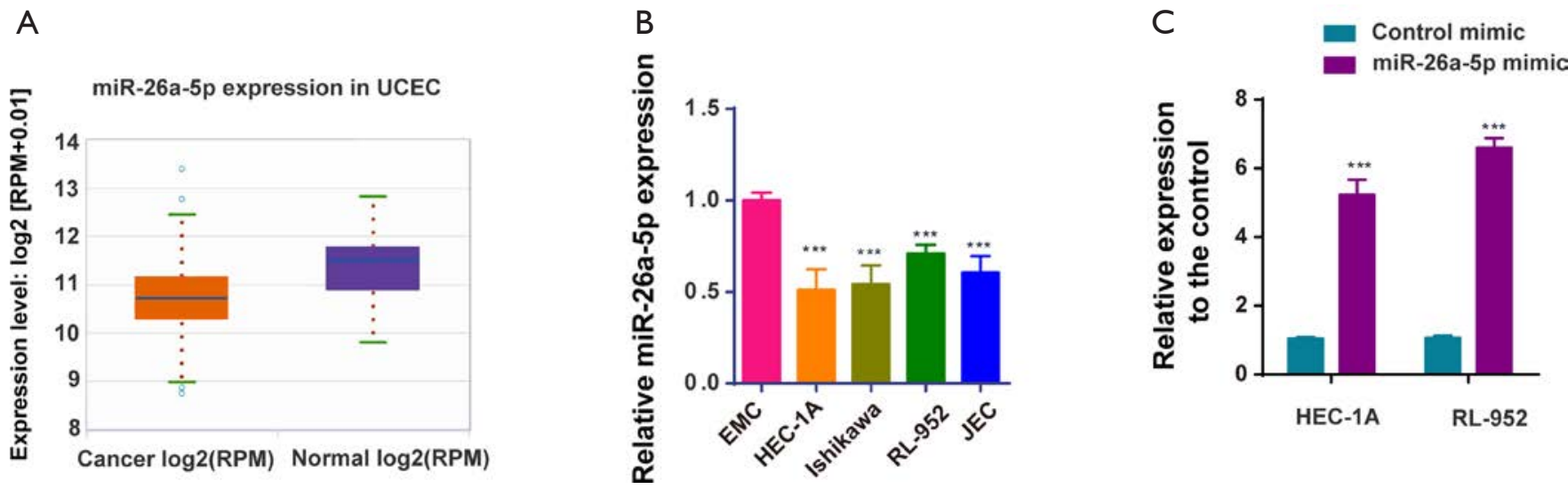

Figure 1 The expression of miR-26a-5p was reduced in endometrial cancer. (A) Differential expression for miR-26a-5p in 538 UCEC samples and 33 normal samples from Starbase 3.0 project. Data were scaled with $\log 2$ (RPM+0.01). (B) Relatively decreased level of miR26a-5p was detected in endometrial cancer cells compared with normal endometrium cells using qRT-PCR normalized to GAPDH. (C) Relative expression of miR-26a-5p was determined by qRT-PCR in HEC-1A and RL-952 cell lines transfected with miR-26a-5p mimic and control mimic. ${ }^{* * *}, \mathrm{P}<0.001$. UCEC, uterine corpus endometrial carcinoma.

\section{cMet was a directly targeting gene of miR-26a-5p in EC cells}

A bioinformatics approach was used to further predict the downstream genes of miR-26a-5p so as to explore the molecular mechanism underlying the inhibitory effect of miR-26a-5p on EC in vivo and in vitro. The results from the starBase website indicated that the mesenchymal-epithelial transition factor (cMet) might be one of the best candidates (Figure $4 A$ ). cMet was a receptor tyrosine kinase with one known ligand named HGF. The hyperactive cMet/HGF pathway played an indispensable role in the occurrence, development, invasion, and metastasis of many tumors, including EC, indicating cMet as a promising checkpoint in the clinical trial. The luciferase reporter assay was used to confirm the correlation between miR-26a-5p and cMet. The result showed that the upregulation of miR-26a-5p significantly reduced the luciferase activity of cMet-wt instead of cMet-mut activity in $293 \mathrm{~T}$ cells, confirming the targeting relationship between miR-26a-5p and cMet (Figure $4 B$ ). The level of cMet mRNA was tested using qRT-PCR assay when transfecting miR-26a-5p into EC cells to validate further that the expression of $\mathrm{cMet}$ was directly regulated by miR-26a-5p. The result revealed that upregulated miR-26a-5p expression dramatically decreased the transcriptional activity of cMet in EC cells (Figure 4C). Moreover, the expression of cMet and other downstream components of the cMet pathway (p-ERK1 and p-Akt) was detected. The findings demonstrated that elevated miR26a-5p expression could suppress the cMet pathway via affecting cMet expression (Figure 4D,E). Taken together, the data indicated that cMet was a directing target of miR26a-5p in EC cells.

\section{MiR-26a-5p suppressed the progression of EC via regulating the cMet patbway}

MiR-26a-5p mimic and cMet vector were co-transfected into EC cells, and cell proliferation was assessed using the CCK-8 assay to confirm further that miR-26a-5p functioned in EC cells through the cMet pathway. The data showed that elevated cMet expression eliminated the inhibitory effect of miR-26a-5p on cell proliferation (Figure $5 A$ ). In addition, the outcomes of the EdU assay were similar to those achieved using the CCK- 8 assay (Figure 5B,C). The cell apoptosis was also detected after co-expressing miR-26a-5p. The result indicated that the number of PI/Annexin V-positive cells was significantly less in the group with the co-transfection of miR-26a-5p and cMet than in the group with the co-transfection of miR26a-5p and empty vector (Figure 5D,E), confirming that the co-expression of cMet could alleviate the function of miR$26 a-5 p$ in the apoptosis of EC cells. Overall the findings suggested that miR-26a-5p suppressed the development of $\mathrm{EC}$ via regulating cMet expression. 

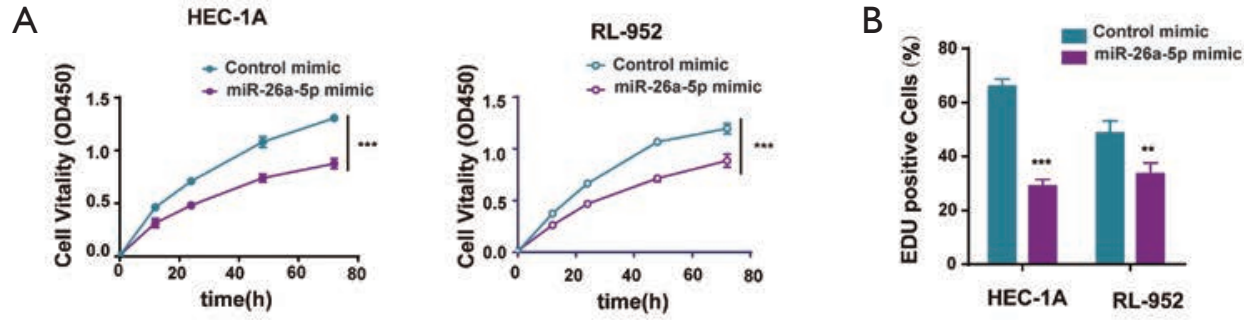

C

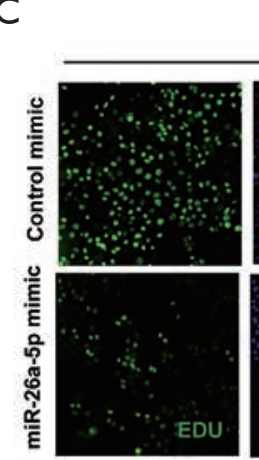

HEC-1A
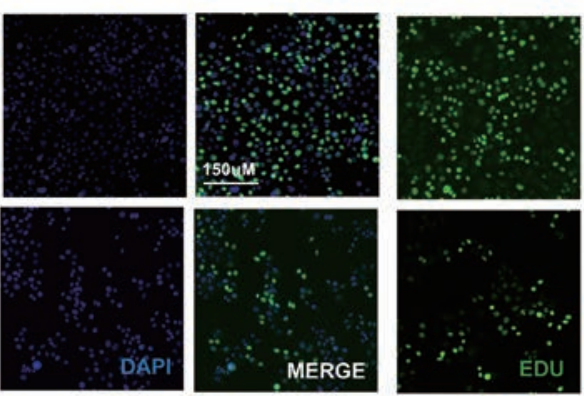

RL-952
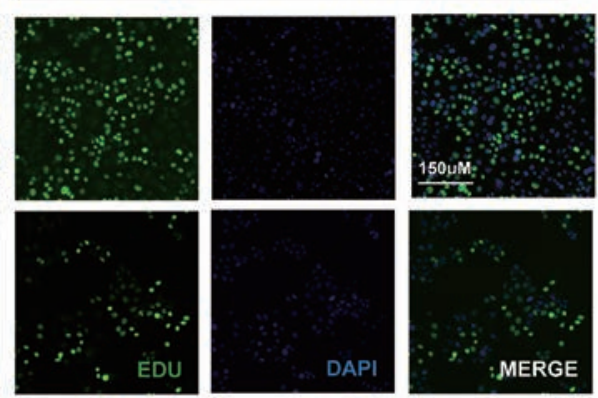

D

HEC-1A
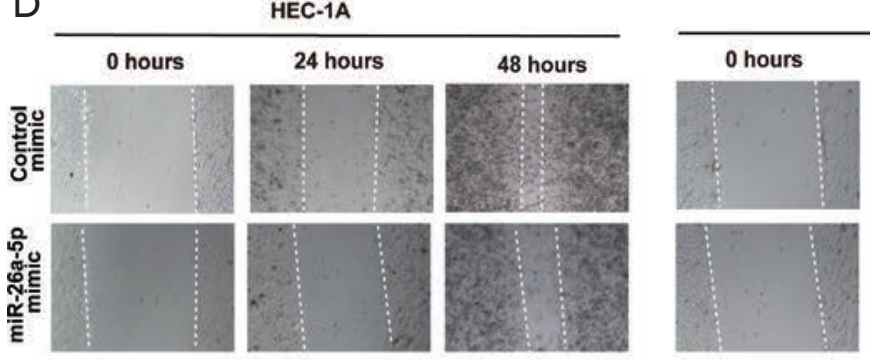

RL-952
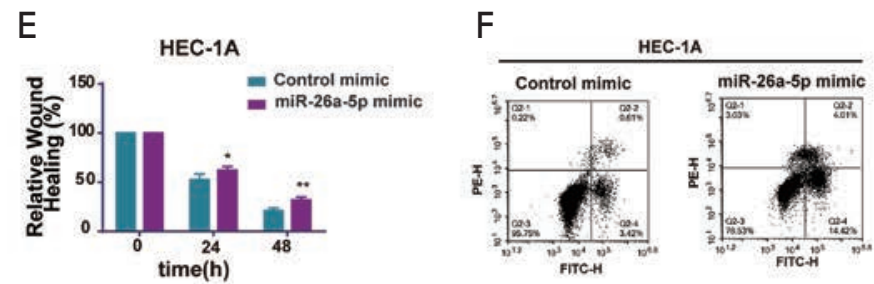

G
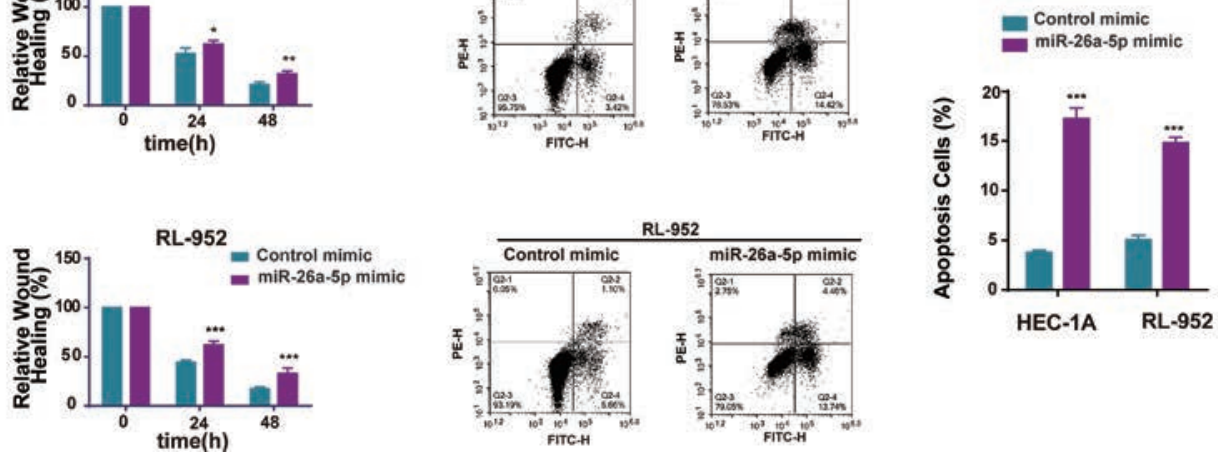

Figure 2 miR-26a-5p inhibited cell proliferation, cell migration and promoted cell apoptosis of EC cells. (A) The inhibitory effect of miR26a-5p on cell vitality of two endometrial cancer cells (HEC-1A and RL-952) was accessed using CCK8 kit. ***, $\mathrm{P}<0.001$. (B,C) EDU staining was applied to measure cell proliferation in EC cells and its statistic graph. ${ }^{* *}, \mathrm{P}<0.01 ;{ }^{* * *}, \mathrm{P}<0.001$. (D,E) Wound healing assay was used to access the ability of cell migration and its statistic graph. ${ }^{*}, \mathrm{P}<0.05$; ${ }^{* *} \mathrm{P}<0.01$; ${ }^{* * *}, \mathrm{P}<0.001$. (F,G) miR-26a-5p was verified to suppress cell apoptosis of HEC-1A and RL-952 cells using flow cytometry. ${ }^{* * *}, \mathrm{P}<0.01$, compared with Control mimic. EC, endometrial cancer; EDU, 5-ethynyl-2'-deoxyuridine. 
A

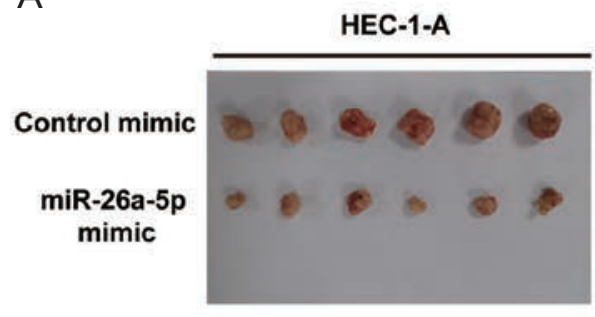

RL-952

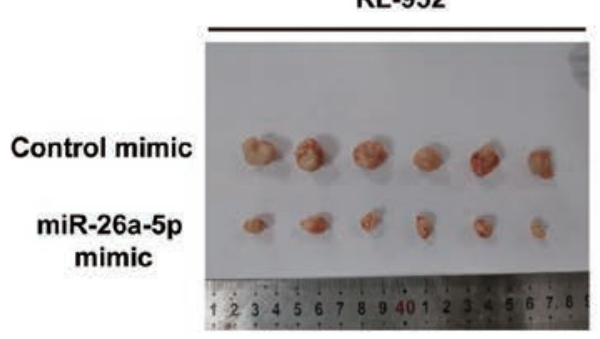

D
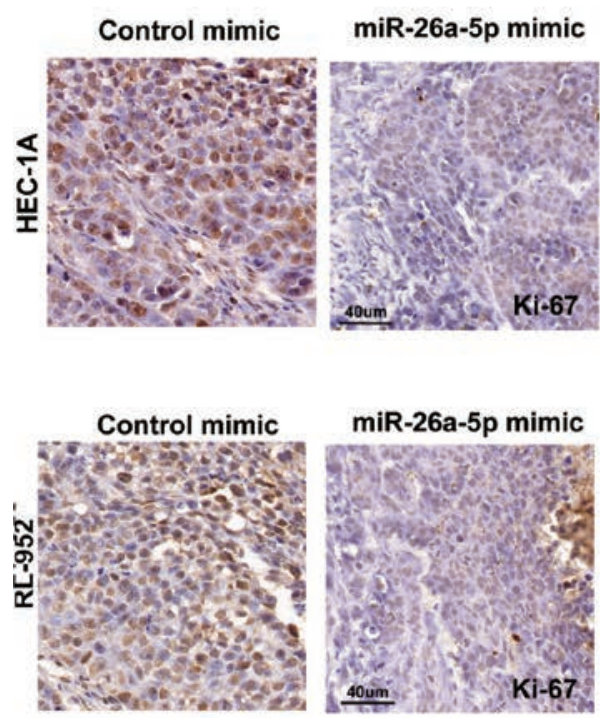

B

HEC-1-A

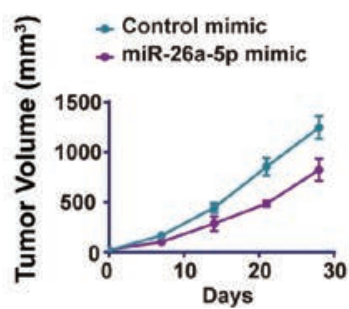

RL-952

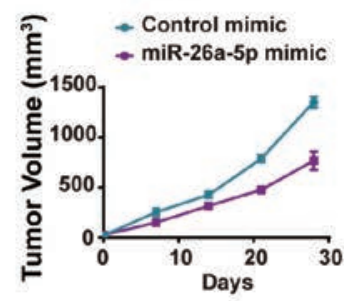

E

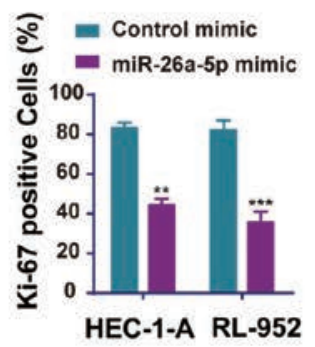

G

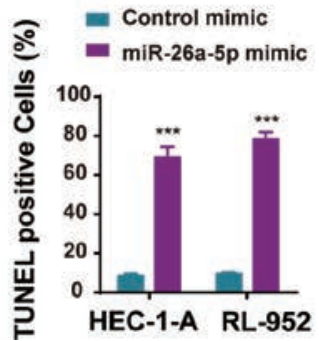

C

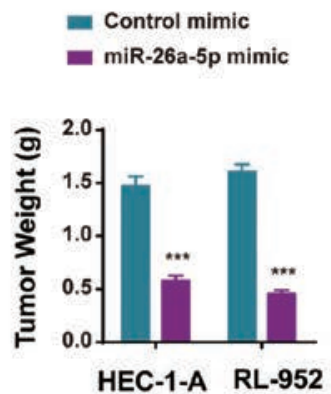

F
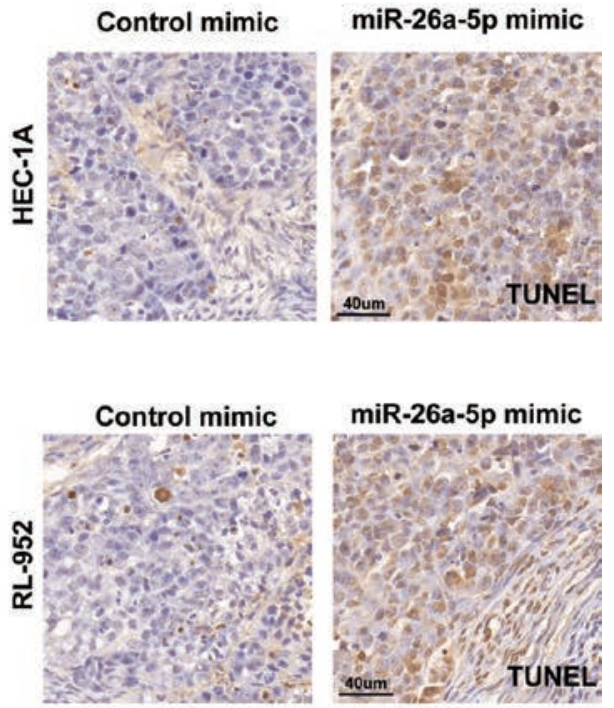

Figure 3 miR-26a-5p acted as one antitumor agent in vivo. (A) Representative pictures of xenografts induced by EC cells transfected with Control mimic and miR-26a-5p mimic after injected into nude mice for 4 weeks. (B,C) Tumor growth curve revealed that upregulation of miR-26a-5p suppressed the growth of transplanted tumors. ${ }^{* *}, \mathrm{P}<0.001$. (D,E) Immunohistochemical staining for Ki-67 in the tumor sample. Scale bar $=40 \mu \mathrm{m} .{ }^{* *}, \mathrm{P}<0.01 ;{ }^{* * *}, \mathrm{P}<0.001$. (F,G) TUNEL staining assay was used to detect cell apoptosis. Scale bar $=40 \mu \mathrm{m}$. ${ }^{* *}$, $\mathrm{P}<0.001$. EC, endometrial cancer. 
A

cMet wt: 5' uaaaaAUCAGGUACCACUUGAu 3'

|| | | | ||| || miR-26a-5p: 3' ucggaUAGGACCUAAUGAACUu 5'

cMet mut: 5' uaaaaUAGAGCUACCUGAACUu 3'
B

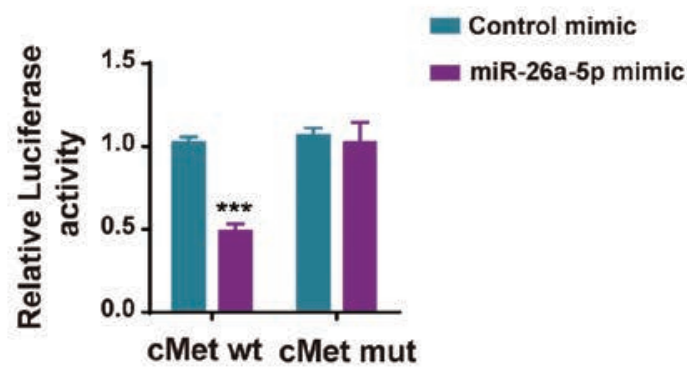

C

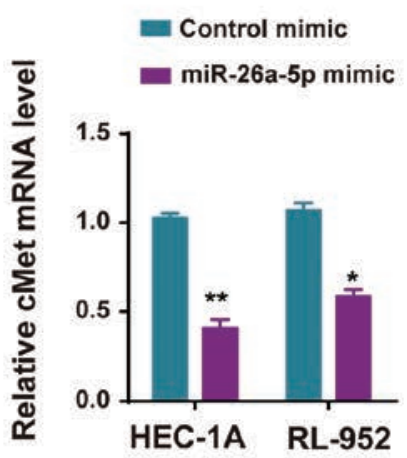

E
Control mimic

miR-26a-5p mimic

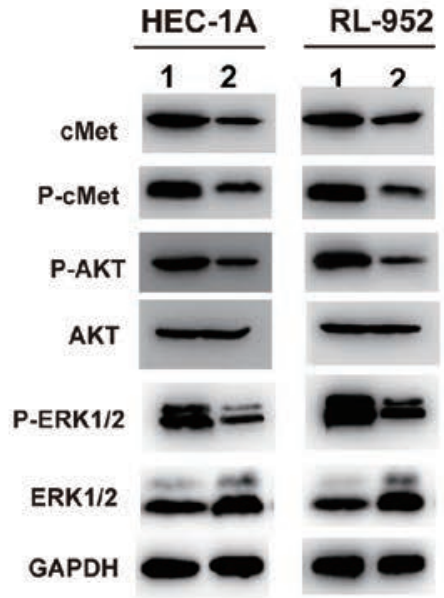

Lane 1: Control mimic

Lane 2: miR-26a-5p mimic

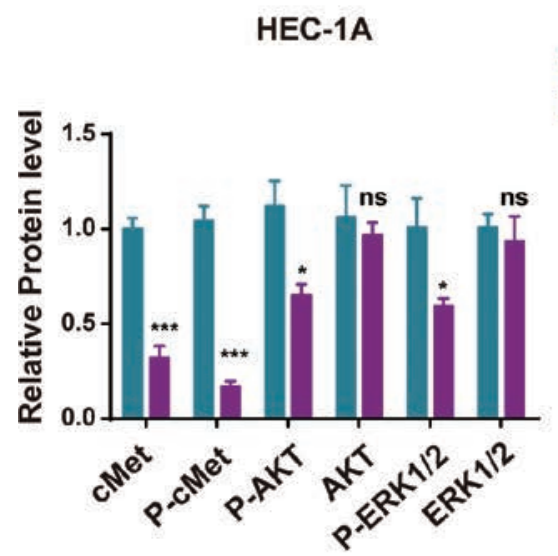

RL952

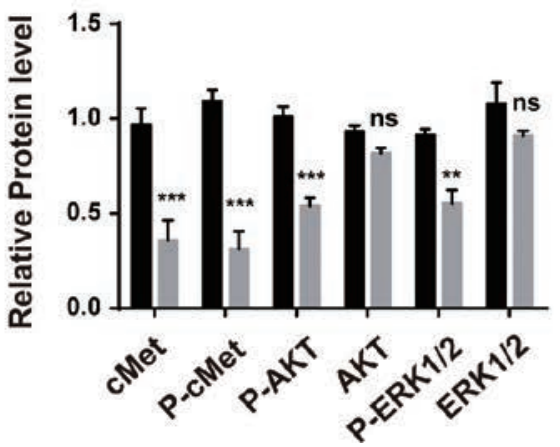

Figure 4 cMet was a directly targeted gene of miR-26a-5p in EC cells. (A) Sequence alignment of miR-26a-5p with the putative binding sites with in the wild-type regions of cMet. (B) Dual-luciferase reporter assay was used to test the targeting relationship between miR-26a$5 \mathrm{p}$ mimic and cMet WT. *** $\mathrm{P}<0.001$. (C) cMet mRNA level was determined using qRT-PCR in EC cells with elevated miR-26a-5p. *, $\mathrm{P}<0.05$; ** $\mathrm{P}<0.01$. (D,E) Western blot analysis of cMet and its downstream genes (P-cMet, P-AKT, AKT, P-ERK1/2, ERK1/2). *, $\mathrm{P}<0.05$; **, $\mathrm{P}<0.01 ;{ }^{* * *}, \mathrm{P}<0.001$. EC, endometrial cancer. 
A

HEC-1A

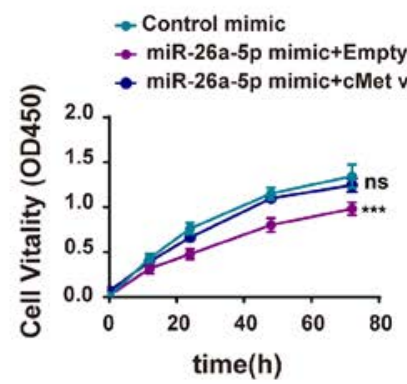

RL-952

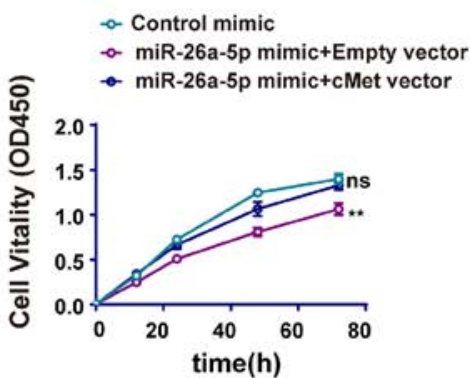

B

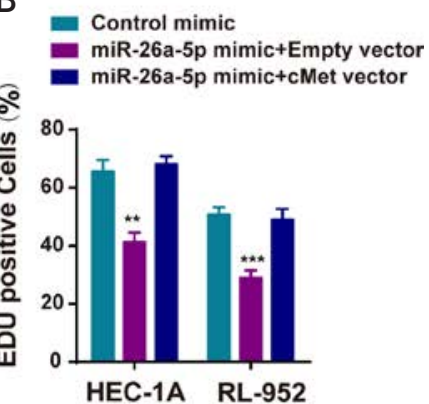

A

HEC-1A

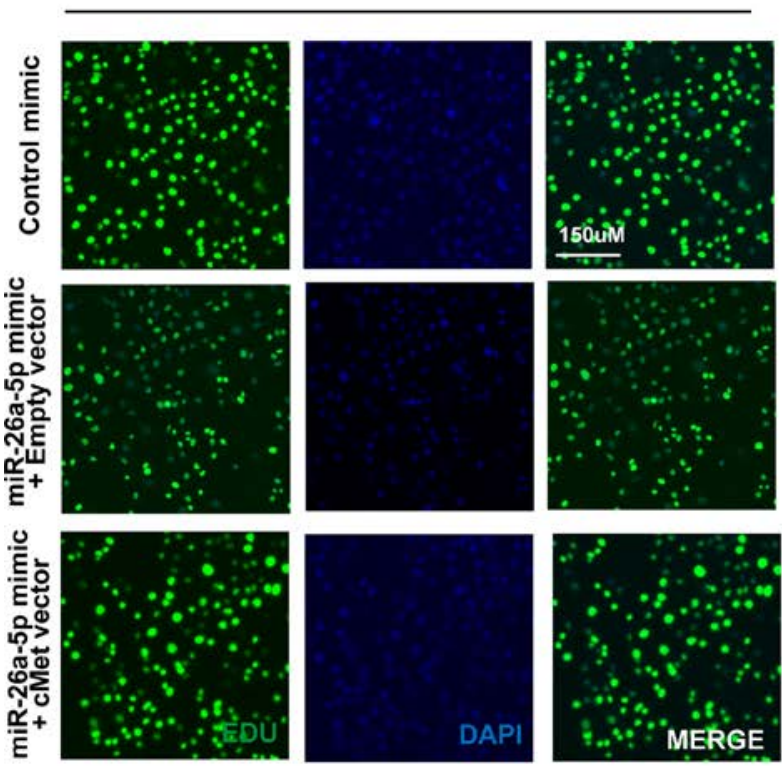

D

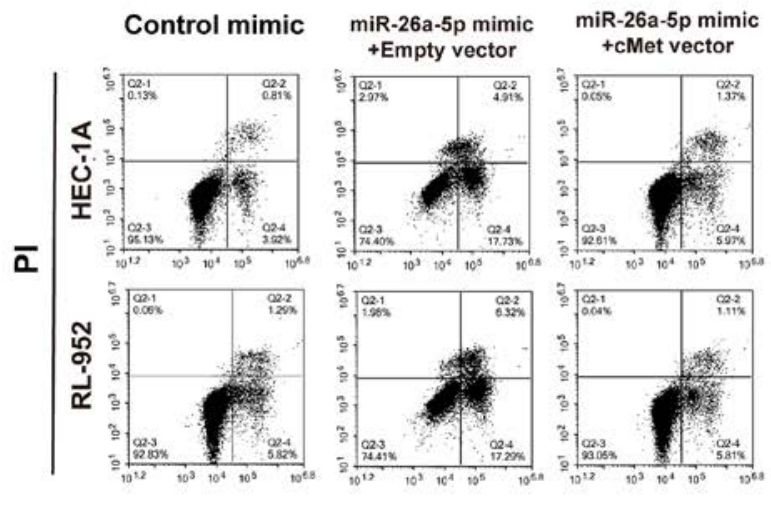

RL-952
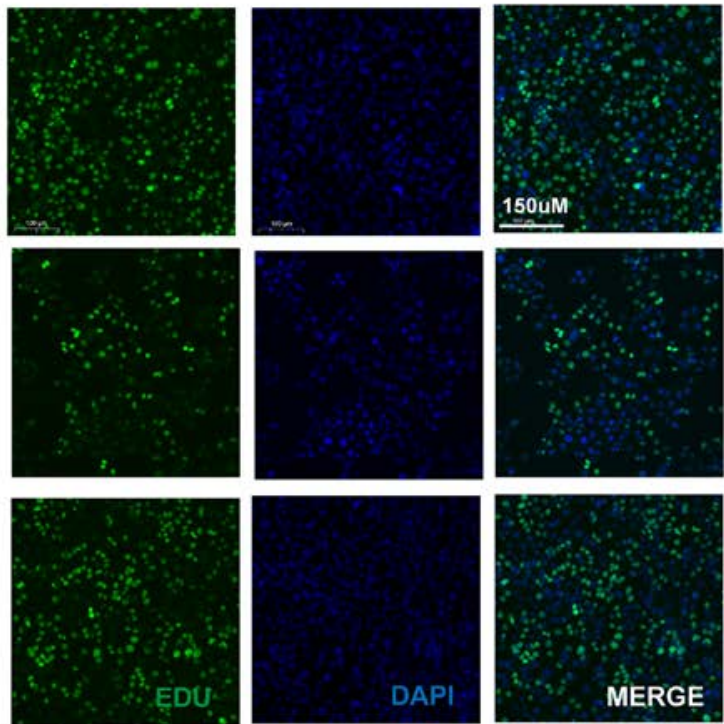

E

- Control mimic

- miR-26a-5p mimic+Empty vector

- miR-26a-5p mimic+cMet vector

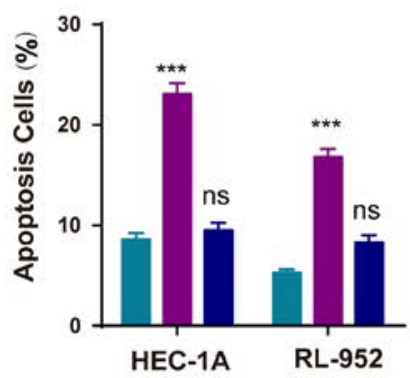

Figure 5 miR-26a-5p suppressed the progression of EC via regulating cMet pathway. (A) Cell vitality assay using CCK8 indicated that cMet overexpression could eliminate the inhibitory effect induced by miR-26a-5p upregulation. **, $\mathrm{P}<0.01$; *** $\mathrm{P}<0.001$. (B,C) Cell proliferation in EC cells and its statistic graph. ${ }^{* *}, \mathrm{P}<0.01$; ${ }^{* *}, \mathrm{P}<0.001$. (D,E) Cell apoptosis was evaluated by flow cytometry through staining $\mathrm{PI}$ and Annexin-V. ${ }^{* * *}, \mathrm{P}<0.001$. EC, endometrial cancer. 


\section{MiR-26a-5p enhanced the effect of cetuximab in EC cells}

Numerous studies reported that cMet was a promising checkpoint for gynecological oncology $(11,21)$. Based on the above study, we learned that miR-26a-5p might be considered as a novel RNAi therapeutic targeting cMet in antitumor activity. Hence, the EGFR pathway interplayed with the cMet pathway, and EGFR-TKIs were found to have antitumor efficacy in EC cells $(29,30)$. Given the interrelationship between EGFR and cMet, it was hypothesized that a combination of cMet target inhibitor with EGFR-TKIs might be a potential approach in EC treatment. For validating the hypothesis, the present study used flow cytometry to explore whether EGFR was expressed on the surface of HEC-1A and RL-952 cells. The result showed that both HEC-1A and RL-952 cells exhibited EGFR expression (Figure 6A). Then, the antitumor effect of cetuximab on EC cells was detected at different dosages. Cetuximab is an anti-EGFR monoclonal antibody with a higher binding affinity for EGFR. The result showed that cetuximab suppressed the growth of EC cells in the CTG assay, and the optimal dose was $20 \mu \mathrm{g} / \mathrm{mL}$ (Figure 6B). Subsequently, cetuximab was added to EC cells at a concentration of $20 \mu \mathrm{g} / \mathrm{mL}$ after transfecting the cells with miR-26a-5p mimic and control mimic with the stimulation factor EGF. The result revealed that cetuximab exhibited a more robust effect on the growth of EC cells with elevated miR-26a-5p levels compared with the control mimic group (Figure $6 C$ ). Moreover, the present study also rechecked the dual inhibition of cMet using miR-26a-5p and EGFR inhibitory antibody cetuximab in vivo. The tumor volume showed a more robust antitumor efficacy of dual agents compared with a single agent (Figure 6D,E). Additionally, no significantly adverse events were observed after drug treatment. In conclusion, the study demonstrated that miR-26a-5p enhanced the inhibitory effect of cetuximab on EC cells, thus suggesting a potential drug combination for the treatment of EC.

\section{Discussion}

The HGF/cMet signaling pathway is hyperactive in various solid tumors, including EC $(12,31)$. The level of HGF significantly increased in patients with endometriosis (32), indicating that the HGF/cMet pathway might be vital in endometrial diseases. In clinical trials, cMet expression in tumors was associated with poor prognosis and resistance to targeted therapy $(7,33)$. Therefore, targeting cMet with small-molecule inhibitors and antibodies was considered a promising strategy in clinical trials (34). Onartuzumab, a one-armed humanized IgG1 antibody, could block the high-affinity binding of HGF to cMet and restrain the activation of downstream signaling pathways after binding to cMet. A phase III study on the use of onartuzumab plus erlotinib in patients with cMet-positive advanced NSCLC was halted because of the lack of clinical efficacy $(35,36)$. The extremely complicated downstream signaling pathway of cMet might be one of main reasons for unsatisfactory clinical outcomes, indicating that Met inhibition via ligandblocking antibodies might not be an effective therapeutic strategy. RNAi interference could regulate cMet expression at the genome level, which might be a novel research strategy for cMet targeting.

The EGFR is important in enhancing HGF-cMETmediated proliferation and invasion of epithelial cells. Given that EGFR and cMet were highly expressed in EC and shared overlapping downstream signaling pathways (37), a drug combination targeting EGFR and cMet might be a predictive approach for the treatment of patients with positive cMet or EGFR TKI resistance. Furthermore, dual checkpoints against cMet and EGFR might reduce the unexpected adverse reactions induced by the feedback loop of monotherapy in clinical therapy. In fact, a bispecific antibody LY3164530 targeting cMet and EGFR has been designed by Lily Pharmaceutical Company. Phase I clinical data indicated that the potential of LY3164530 could not be maximized due to the limitation of fixed stoichiometry (38).

Considering the resistance and the crosstalk that occurs between cMet and EGFR, a novel therapeutic approach using RNAi targeting cMet and monoclonal antibody cetuximab against EGFR was explored in this study. The study was novel in demonstrating that miR-26a-5p was involved in the progression of EC, including cell migration, cell proliferation, and cell apoptosis in vivo and in vitro. Furthermore, miR-26a-5p could regulate the expression of cMet and the downstream HGF/cMet pathway, thus exerting an effect on EC cells. In addition, the upregulation of miR-26a-5p could enhance the inhibitory effect of cetuximab on EC cells compared with using cetuximab alone. The dual therapy using RNA interference and antibody blocking could adjust the stoichiometry of miR- 


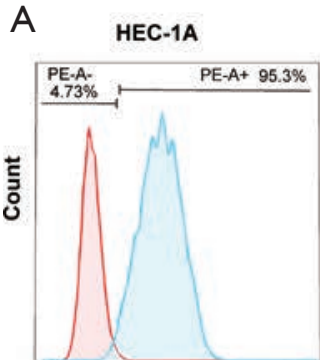

PE-A

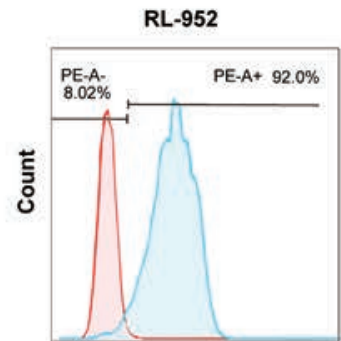

PE-A
C

HEC-1A

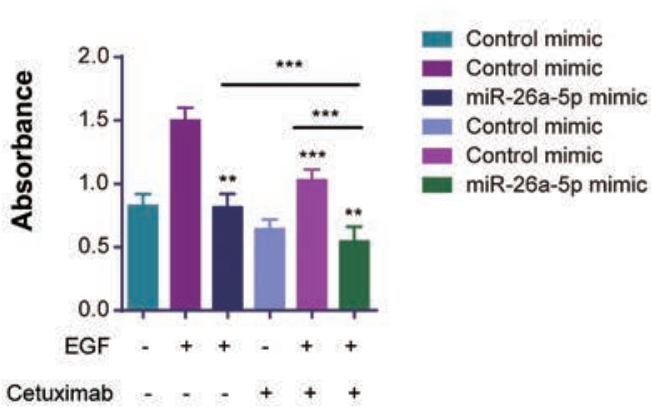

D

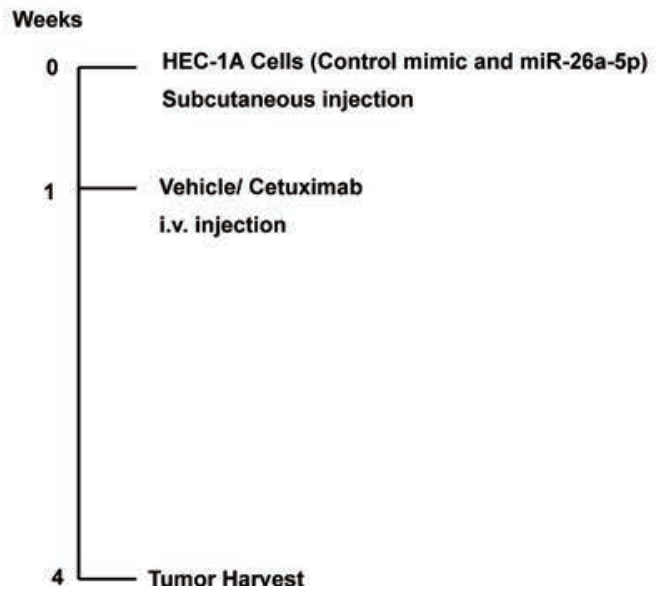

B

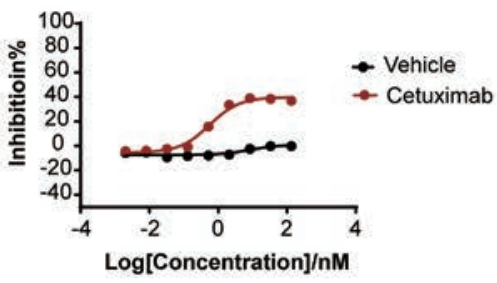

RL-952

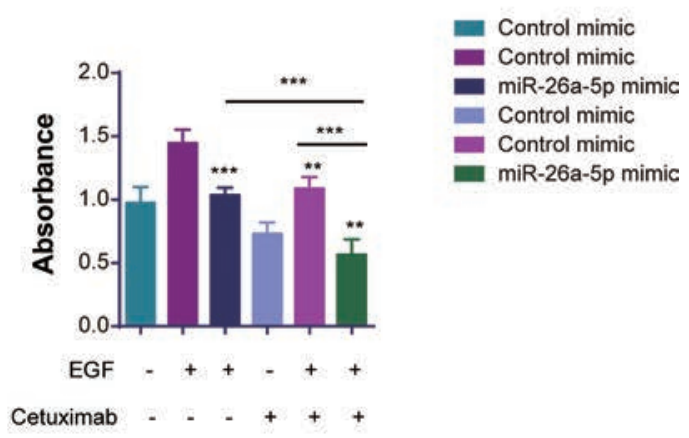

$E$

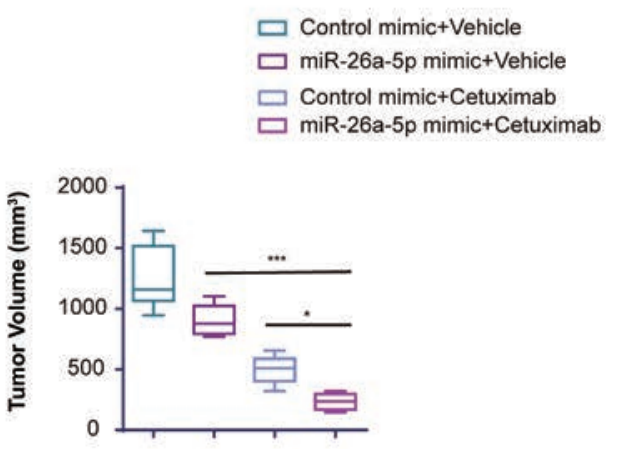

Figure 6 miR-26a-5p could enhance the efficiency of cetuximab in EC cells. (A) Flow cytometry result exhibited the positive EGFR expression on the EC cells. (B) CTG assay indicated cetuximab inhibited the proliferation of HEC-A cells compared with vesicle group. (C) CCK8 assay was applied to detect the inhibitory effect of cetuximab on EC cells with miR-26a-5p overexpression. (D) Representative images of tumors derived from HEC-1A cells subcutaneously injected into Balb/c nude mice at 3 weeks after treatment with cetuximab at $10 \mathrm{mg} / \mathrm{kg}$. (E) Tumor volume graph exhibited the xenograft tumor volumes in Balb/c nude mice treated with dual regent miR-26a-5p and cetuximab. *, $\mathrm{P}<0.05 ;{ }^{* *}, \mathrm{P}<0.01 ;{ }^{* * *}, \mathrm{P}<0.001$. EC, endometrial cancer; EGFR, epidermal growth factor receptor. 
$26 a-5 p$ and cetuximab, thus maximizing the potential and providing a novel approach for EC treatment.

\section{Conclusions}

In this study, miR-26a-5p was demonstrated to exhibit abnormal expression profile in EC cells. Meanwhile, the reduced miR-26a-5p was associated with cell proliferation, migration and apoptosis of EC cells. Additionally, the anti-tumor activity of miR-26a-5p was also found in the xenograft model. The relationship between miR-26a-5p and its direct target cMet was predicted and demonstrated in EC cells. Notably, miR-26a-5p enhanced the inhibitory effect of cetuximab on EC cells, providing a promising clinical strategy for the treatment of EC.

\section{Acknowledgments}

Funding: This work was supported by the Beijing Natural Science Foundation (7184211); National Natural Science Foundation of China (81801402); Beijing Natural Science Foundation Program and Scientific Research Key Program of Beijing Municipal Commission of Education (KM201610025023), and the Beijing key clinical specialty project.

\section{Footnote}

Reporting Checklist: The authors have completed the ARRIVE reporting checklist. Available at http://dx.doi. org/10.21037/atm-20-3166

Data Sharing Statement: Available at http://dx.doi. org/10.21037/atm-20-3166

Conflicts of Interest: All authors have completed the ICMJE uniform disclosure form (available at http://dx.doi. org/10.21037/atm-20-3166). The authors have no conflicts of interest to declare.

Ethical Statement: The authors are accountable for all aspects of the work in ensuring that questions related to the accuracy or integrity of any part of the work are appropriately investigated and resolved. The experiments were performed according to the USA National Institutes of Health guidelines and approved by the local animal care and use committee of Capital Medical University (18-2032).
Open Access Statement: This is an Open Access article distributed in accordance with the Creative Commons Attribution-NonCommercial-NoDerivs 4.0 International License (CC BY-NC-ND 4.0), which permits the noncommercial replication and distribution of the article with the strict proviso that no changes or edits are made and the original work is properly cited (including links to both the formal publication through the relevant DOI and the license). See: https://creativecommons.org/licenses/by-nc-nd/4.0/.

\section{References}

1. Jemal A, Bray F, Center MM, et al. Global cancer statistics. CA Cancer J Clin 2011;61:69-90.

2. McAlpine JN, Temkin SM, Mackay HJ. Endometrial cancer: Not your grandmother's cancer. Cancer 2016;122:2787-98.

3. Lax SF, Kurman RJ. A dualistic model for endometrial carcinogenesis based on immunohistochemical and molecular genetic analyses. Verh Dtsch Ges Pathol 1997;81:228-32.

4. Prat J. Pathology of cancers of the female genital tract. Int J Gynaecol Obstet 2015;131 Suppl 2:S132-45.

5. Lee YC, Lheureux S, Oza AM. Treatment strategies for endometrial cancer: current practice and perspective. Curr Opin Obstet Gynecol 2017;29:47-58.

6. Amant F, Moerman P, Neven P, et al. Treatment modalities in endometrial cancer. Curr Opin Oncol 2007;19:479-85.

7. Rashed WM. C-MET as a potential target therapy toward personalized therapy in some pediatric tumors: An overview. Crit Rev Oncol Hematol 2018;131:7-15.

8. Engelman JA, Zejnullahu K, Mitsudomi T, et al. MET amplification leads to gefitinib resistance in lung cancer by activating ERBB3 signaling. Science 2007;316:1039-43.

9. Prat M, Narsimhan RP, Crepaldi T, et al. The receptor encoded by the human c-MET oncogene is expressed in hepatocytes, epithelial cells and solid tumors. Int J Cancer 1991;49:323-8.

10. Di Renzo MF, Olivero M, Ferro S, et al. Overexpression of the c-MET/HGF receptor gene in human thyroid carcinomas. Oncogene 1992;7:2549-53.

11. Li M, Xin X, Wu T, et al. HGF and c-Met in pathogenesis of endometrial carcinoma. Front Biosci (Landmark Ed) 2015;20:635-43.

12. KhoshdelRad N, Salehi Z, Mashayekhi F, et al. Soluble c-Met expression in the peritoneal fluid and serum of patients with different stages of endometriosis. Arch 
Gynecol Obstet 2014;289:1107-12.

13. Linklater ES, Tovar EA, Essenburg CJ, et al. Targeting MET and EGFR crosstalk signaling in triple-negative breast cancers. Oncotarget 2016;7:69903-15.

14. Pietraszek-Gremplewicz K, Simiczyjew A, Dratkiewicz E, et al. Expression level of EGFR and MET receptors regulates invasiveness of melanoma cells. J Cell Mol Med 2019;23:8453-63.

15. Simiczyjew A, Dratkiewicz E, Van Troys M, et al. Combination of EGFR Inhibitor Lapatinib and MET Inhibitor Foretinib Inhibits Migration of Triple Negative Breast Cancer Cell Lines. Cancers (Basel) 2018;10.

16. Dratkiewicz E, Simiczyjew A, Pietraszek-Gremplewicz K, et al. Characterization of Melanoma Cell Lines Resistant to Vemurafenib and Evaluation of Their Responsiveness to EGFR- and MET-Inhibitor Treatment. Int J Mol Sci 2019;21.

17. Ejskjaer K, Sorensen BS, Poulsen SS, et al. Expression of the epidermal growth factor system in endometrioid endometrial cancer. Gynecol Oncol 2007;104:158-67.

18. Nishimura T, Nakamura K, Yamashita S, et al. Effect of the molecular targeted drug, erlotinib, against endometrial cancer expressing high levels of epidermal growth factor receptor. BMC Cancer 2015;15:957.

19. Khalifa MA, Mannel RS, Haraway SD, et al. Expression of EGFR, HER-2/neu, P53, and PCNA in endometrioid, serous papillary, and clear cell endometrial adenocarcinomas. Gynecol Oncol 1994;53:84-92.

20. Niikura H, Sasano H, Matsunaga G, et al. Prognostic value of epidermal growth factor receptor expression in endometrioid endometrial carcinoma. Hum Pathol 1995;26:892-6.

21. Thoury A, Descatoire V, Kotelevets L, et al. Evidence for different expression profiles for c-Met, EGFR, PTEN and the mTOR pathway in low and high grade endometrial carcinomas in a cohort of consecutive women. Occurrence of PIK3CA and K-Ras mutations and microsatellite instability. Histol Histopathol 2014;29:1455-66.

22. Migliore C, Petrelli A, Ghiso E, et al. MicroRNAs impair MET-mediated invasive growth. Cancer Res 2008;68:10128-36.

23. Giglio S, Vecchione A. c-Met and miRs in Cancer. Biomedicines 2015;3:32-44.

24. Jayaraman M, Radhakrishnan R, Mathews CA, et al. Identification of novel diagnostic and prognostic miRNA signatures in endometrial cancer. Genes Cancer
2017;8:566-76.

25. Pan-Cancer Differential Expression Analysis of microRNA Genes across 32 types of Cancers. Available online: http:// starbase.sysu.edu.cn/panMirDiffExp.php

26. Salic A, Mitchison TJ. A chemical method for fast and sensitive detection of DNA synthesis in vivo. Proc Natl Acad Sci U S A 2008;105:2415-20.

27. Limsirichaikul S, Niimi A, Fawcett H, et al. A rapid nonradioactive technique for measurement of repair synthesis in primary human fibroblasts by incorporation of ethynyl deoxyuridine (EdU). Nucleic Acids Res 2009;37:e31.

28. Kotogany E, Dudits D, Horvath GV, et al. A rapid and robust assay for detection of S-phase cell cycle progression in plant cells and tissues by using ethynyl deoxyuridine. Plant Methods 2010;6:5.

29. Takahashi K, Saga Y, Mizukami H, et al. Cetuximab inhibits growth, peritoneal dissemination, and lymph node and lung metastasis of endometrial cancer, and prolongs host survival. Int J Oncol 2009;35:725-9.

30. Moinfar F, Gogg-Kamerer M, Sommersacher A, et al. Endometrial stromal sarcomas frequently express epidermal growth factor receptor (EGFR, HER-1): potential basis for a new therapeutic approach. Am J Surg Pathol 2005;29:485-9.

31. Markowska A, Szarszewska M, Zurawski J, et al. Studies on selected molecular factors in endometrial cancers. Adv Clin Exp Med 2018;27:1417-24.

32. Bradford MM. A rapid and sensitive method for the quantitation of microgram quantities of protein utilizing the principle of protein-dye binding. Anal Biochem 1976;72:248-54.

33. Pilotto S, Carbognin L, Karachaliou N, et al. Tracking MET de-addiction in lung cancer: A road towards the oncogenic target. Cancer Treat Rev 2017;60:1-11.

34. Van Der Steen N, Giovannetti E, Pauwels P, et al. cMET Exon 14 Skipping: From the Structure to the Clinic. J Thorac Oncol 2016;11:1423-32.

35. Spigel DR, Edelman MJ, O'Byrne K, et al. Results From the Phase III Randomized Trial of Onartuzumab Plus Erlotinib Versus Erlotinib in Previously Treated Stage IIIB or IV Non-Small-Cell Lung Cancer: METLung. J Clin Oncol 2017;35:412-20.

36. Kishi K, Sakai H, Seto T, et al. First-line onartuzumab plus erlotinib treatment for patients with MET-positive and EGFR mutation-positive non-small-cell lung cancer. Cancer Treat Res Commun 2019;18:100113. 
37. Shin SY, Muller AK, Verma N, et al. Systems modelling of the EGFR-PYK2-c-Met interaction network predicts and prioritizes synergistic drug combinations for triple-negative breast cancer. PLoS Comput Biol 2018;14:e1006192.

Cite this article as: Liu Y, Cai Y, Chang Y. Dual inhibition of RNAi therapeutic miR-26a-5p targeting cMet and immunotherapy against EGFR in endometrial cancer treatment. Ann Transl Med 2021;9(1):5. doi: 10.21037/atm-20-3166
38. Patnaik A, Gordon M, Tsai F, et al. A phase I study of LY3164530, a bispecific antibody targeting MET and EGFR, in patients with advanced or metastatic cancer. Cancer Chemother Pharmacol 2018;82:407-18. 